\title{
Conduction of reparative dentin: A pulp protecting approach by indirect pulp capping in deep carious lesion with biodentine
}

\author{
Rafeza Sultana and Md. Shamsul Alam
}

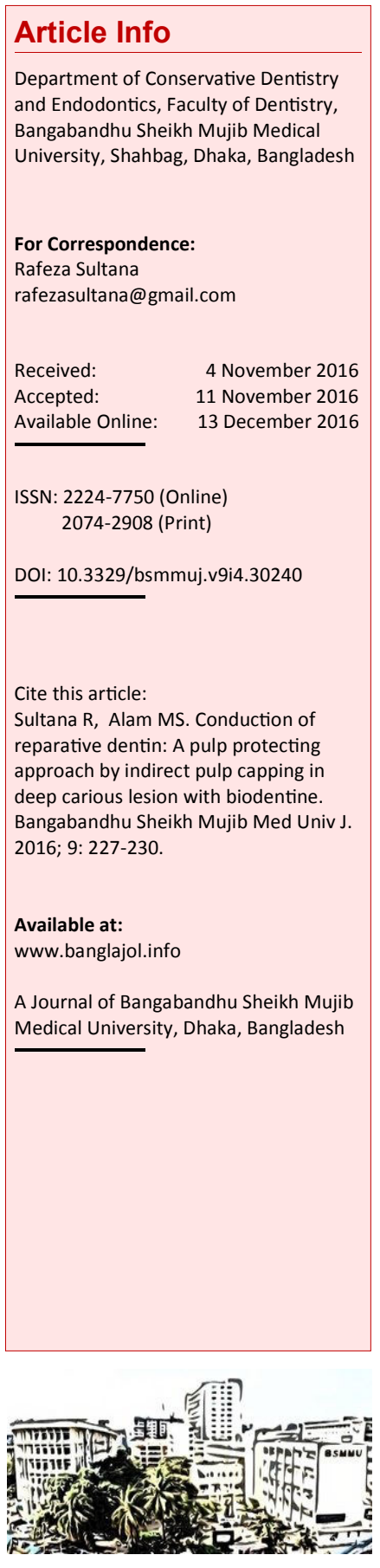

\section{Abstract}

This case report represents, the treatment of deep carious lesion in a male was done by using indirect pulp capping with biodentine. The clinical and radiological outcomes were evaluated. The patient was presented with mild to moderate sensitivity on thermal (cold) stimulation and discomfort associated with eating in the left mandibular first molar. The clinico-radiographical examination revealed a disto-occlusal carious lesion, very close to the pulp, absence of radiolucencies in the periapical region and no periodontal space widening. Pulp sensitivity was confirmed by thermal pulp vitality test. Indirect pulp capping treatment was performed with biodentine. Following 6 months of the treatment, the clinical symptoms were resolved and a calcific bridge was found at the dentin over the pulp and biodentine interface that was indicative the evidence of reparative dentin formation.

\section{Introduction}

Pulp and dentin encompass an intimate complex in which bacterial contamination of the dentin frankly affects the pulp. A mild carious invasion even incurs a pulpal reaction. If the etiology is removed early, healing occurs near the begining. 1 Dental caries is the prime threat to the heath of the dental pulp that causes progressive damage of the tooth structure. The damage can convert the reversible pulp to irreversible state. In deliberation of conservative dentistry, it can be considered as preventive or interceptive endodontics that allows a compromized pulp to recover and protect pulp from further damage. Indirect pulp capping is one of the approaches to protect the pulp of teeth with deep carious lesion. In indirect pulp capping technique, following removal of infected, soft carious dentin, demineralized (affected or sterile dentin) is needed to be left which can be remineralized by using pulp capping therapeutics. 2

An imperative precedence in the treatment of deep carious lesions is to preserve pulp vitality. .3 The conservative pulp management has the potentiality to reduce the need for a more invasive treatment. 4 However, after pulp exposure, such conservative treatment is dubious and unpredictable. In addition, during caries removal if the pulp is exposed, successful outcome will be significantly reduced. 5 In order to avoid pulp exposure in such case, a conservative procedure has been recommended, the indirect pulp treatment (IPT). $\underline{6}$

Retrospective and prospective studies showed that in both dentitions the success rate for indirect pulp treatment was similar, ranging from 73 to $93 \%$ after 2 weeks to 11 years of follow-up. $?$

The search for the ideal vital pulp materials the researchers has lead to investigate different new innovative therapeutics for successful pulp capping such as $\mathrm{Ca}(\mathrm{OH})_{2}$, $\mathrm{ZnO}$ eugenol cement, polycarboxylate cement, glass ionomer cement, antibiotic, growth factor, emdogain, bioglass, MTA and recently biodentine. $\underline{8}$

Calcium hydroxide is considered as widely used pulp capping agent. However, it does not provide close adaptation to dentin, gradual degradation, develop inadequate reparative dentin with tunnel defect which provide path way for penetration of microorganisms.?

New bioactive cement, biodentine ${ }^{\mathrm{TM}}$ (Septodont, St. Maur- des- Fosses France), was recently launched in the dental market as a dentin replacement. Biodentine ${ }^{\mathrm{TM}}$ consists of a powder in a capsule and liquid in a pipette. The powder mainly contains tri-calcium silicate as a main core material, di-calcium silicate as second core material, calcium carbonate and oxide as fillers, iron oxide as shade and zirconium oxide as radiopacifier. The liquid 
consists of $\mathrm{CaCl}_{2}$ as accelator and hydrosoluble polymer as water reducing agents. The powder is mixed with the liquid in a capsule in the amalgamator for $30 \mathrm{sec}$. Setting time of biodentine ${ }^{\mathrm{TM}}$ is about $12 \mathrm{~min}$. It can be used in both in crown and root. Its crown uses include temporary closer, cervical filling, indirect and direct pulp capping and pulpotomy. On root, it is in repair of floor perforation, internal and external resorption and retrograde root canal obturation. $\underline{10}$

In this case report, indirect pulp capping with biodentine was performed to maintain pulp vitality by conduction of reparative dentin over the pulp.

\section{Case Report}

A 20 year old patient reported to the Department of Conservative Dentistry and Endodontics, BSMMU, Dhaka with the complaints of mild to moderate sensitivity on thermal (cold) stimulation and discomfort associated with eating in the left mandibular first molar. The clinical examination revealed a deep disto-occlusal carious lesion in the mandibular left first molar tooth without pulp exposure. The tooth was responded normally to percussion and palpation. Normal appearance of adjacent gingival tissue was evident. The radiograph revealed a deep distal carious lesion, very close to the pulp, absence of radiolucencies in the periapical region and no periodontal space widening. Pulp sensitivity was confirmed by thermal (cold test) pulp vitality test. There was no exaggerated and lingering pain and quickly disappear after stimulus was removed.
A diagnosis of reversible pulpitis of left mandibular first molar was made and the treatment plan was established to preserve the pulp vitality by indirect pulp capping with biodentine followed by permanent restoration with composite resin.

The whole treatment procedure was explained to the patient and consent was taken. After mouth preparation local anesthesia was administered and isolation was done with cotton roll. Firstly, surrounding caries was removed by using no. \#4 diamond bur at high speed with sufficient water spray. Infected dentin from cavity walls was removed with a spoon excavator followed by low speed round carbide bur compatible with the size of the cavity very carefully leaving the affected dentin to avoid the pulp exposure. The cavity was flashed with normal saline and dried with sterile cotton pellets. Biodentine ${ }^{\mathrm{TM}}$ (septodont) powder and liquid was mixed according to manufacturer recommendations in an automatic mixture (amalgamator) for $30 \mathrm{sec}$. Then putty like consistency was dispensed on to a mixing pad and was applied to the cavity up to $3 \mathrm{~mm}$ thickness by amalgam carrier. About $12 \mathrm{~min}$ after mixing, the material was set hard and the tooth was restored with composite restoration (giomer) to replace enamel. Occlusion was checked for any high spot. Then post operative radiograph was taken as base line radiograph. The patient was recalled for clinical and radiological evaluation at 3, 6 months interval. At 6 months, the radiographic evaluation (Figure 2,3 ) showed a consistent reparative dentin formation at the dentin over the pulp and biodentine interface.

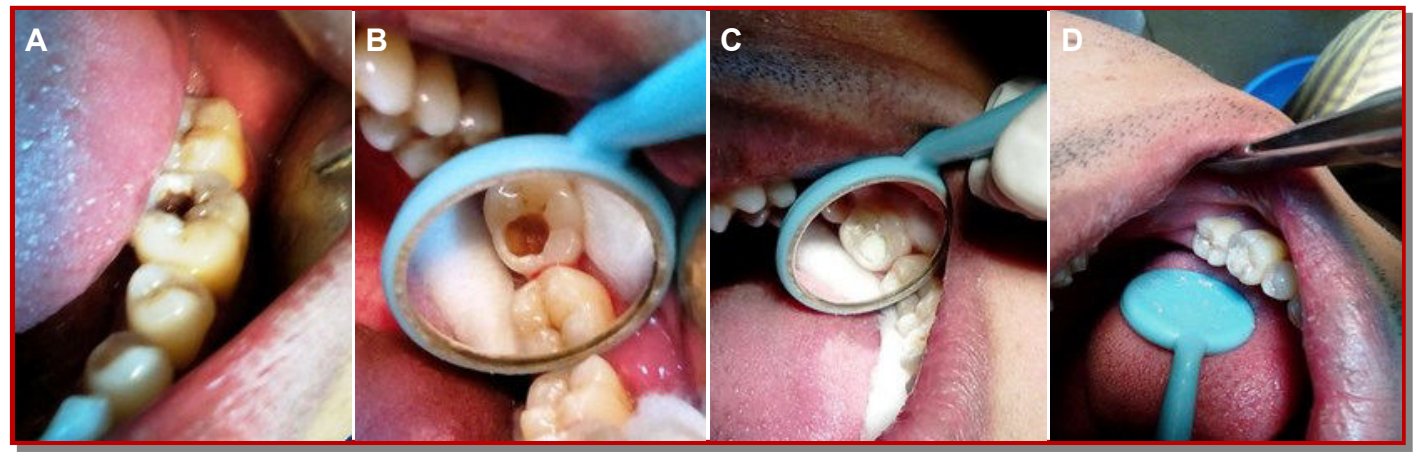

Figure 1: Steps of clinical procedure: (A) Deep caries in 36 No. tooth, (B) After removal of caries, (C) Placement of biodentine, (D) Permanent restoration with composite resin

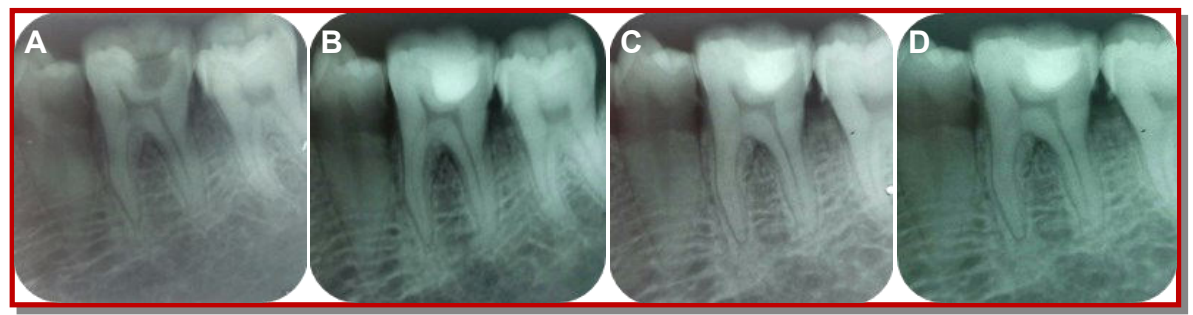

Figure 2: Intra oral periapical radiographs at (A) initial, (B) the base line, (C) 3 months, (D) 6 months intervals 


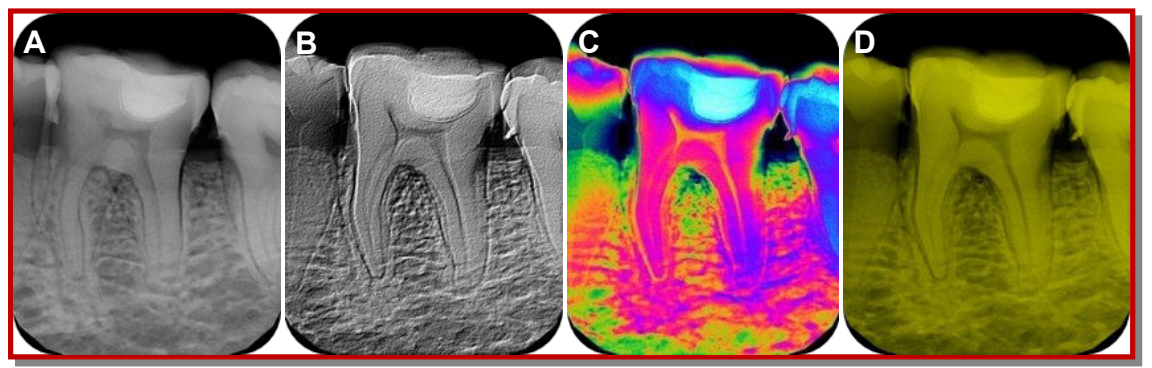

Figure 3: Radio visiography (RVG) of IPC 6 months follow-up periods

\section{Discussion}

The choice for indirect pulp capping treatment in this case was based on careful pulp diagnosis, which was supported by evaluation of the history of pain and clinical and radiographic findings. Most of the studies reported in the literature evaluated IPC for the treatment of deep carious lesion in primary molars while some clinical studies were carried out on the permanent teeth. Active carious lesion can be distinguished into two layers; infected dentin which is a superficial soft layer of collagen fibrils was degraded that cannot be remineralized; and affected dentin, which is partially demineralized, with intact collagen fibrils that can be reminaralized. However the clinical recognition of these two layers is a difficult task. Experience and clinical judgment are essential skills for appropriate removal of infected dentin, leaving the potentially reminaralized affected dentine. Some authors stated that the complete removal of decay is not essential in a second step. Their clinical and radiological results support the theory that the decay process was apparently arrested after indirect pulp capping with a single visit procedure.11 Fairbourn et al reported that the second appointment may not be necessary if the final restoration maintains the cavity seal and the tooth remained asymptomatic. 12

This case report demonstrates that stepwise excavation is not always necessary. The newer calcium silicate cement, biodentine may be especially useful in achieving even greater success in these cases.

The age of the patient is another factor to be considered since conservative treatments have been more indicated for young patients. In this case study, the patient was 20 years old. It is known that there is a reduction in the cell population, the pulpal volume and also the vascular supply with the increase of pulp age. $\underline{13}$

The prognosis of direct pulp capped teeth is much reduced in comparison with indirect pulp capped teeth. The success of the direct pulp capped teeth is $37 \%$ after 5 years and $13 \%$ after 10 years. This result compares with $86 \%$ rate of success over 10 years, in indirect pulp capped teeth. These studies make obvious the significance of avoiding pulp exposure. $\underline{14}$

The material of choice to be placed over the demineralized dentin is another issue of discussion in this case report.

Calcium hydroxide has been used as lining material since $1920_{\mathrm{s}}$. Because of its' high alkaline $\mathrm{pH}$ is about 12 , that can neutralize the acidic bacterial byproduct. The high $\mathrm{pH}$ creates an environment conductive to formation of reparative dentin. In addition, $\mathrm{Ca}(\mathrm{OH})_{2}$ has the capacity to mobilize the growth factors from the dentin matrix, causing the formation of new dentin. $\underline{.15,16}$ Unfortunately, self setting $\mathrm{Ca}(\mathrm{OH})_{2}$ liners are highly soluble and subjected to dissolution over time.16 Traditional Ca $(\mathrm{OH})_{2}$ liners are easily lost during acid etching. Dentin bonding agents that contain water or alcohol can also detrimentally affect the properties of $\mathrm{Ca}$ $(\mathrm{OH})_{2} \cdot \underline{.17}$

Adhesive resin can be acidic that may cause pulpal irritation. Many dentin bonding agents and resin reinforced glass ionomer are actually unfavorable to pulp tissue. Owing to certain disadvantages of those materials, other alternatives have been recommended. $\underline{18}$

One such alternative is Mineral Trioxide Aggregates (MTA); initially developed as a root repair material. It has been used for pulp capping as an eligible replacement for the calcium hydroxide based materials, as it has demonstrated a promising clinical outcome. However, MTA is also overwhelmed with problems like technique sensitive i.e. difficult to manipulate, longer initial setting time ( 2 hours 45 min), difficult to remove after setting, no known solvent for the material, discoloration potentiality, expensive as well. $\underline{19}$

To beat these drawbacks, one among the recently developed biomaterials is biodentine (Septodont, France); based on calcium silicate system can be a comparable replacement for MTA. It is touted to be "Dentin in a Capsule", because of its outstanding biocompatibility, physical properties and bonding to the dental hard tissues. $\underline{20}$

Calcium hydroxide ions are released from the cement during the setting phase of Biodentin. This results in a $\mathrm{pH}$ of about 12.5 that may cause the basification of the surroundings. This high $\mathrm{pH}$ inhibits the augmentation of micro organisms and can disinfect the dentin. $\underline{21}$

Biodentine can adhere to tooth surface by micromechanical adhesion. It is also recommended that there might a possible ion exchange contributing to further adhesion of the cement giving terrific resistance to microleakage and bacterial infiltration. 22

Another recently introduced material is TheraCal 
LC (2012). It is in simplistic chemical entry, a material that offers the pulpal or dentin benefits of both calcium hydroxide and resin modified glass ionomer in one product. The material demonstrates strong physical properties with low water solubility and can be light cured up to a thickness of $1.0 \mathrm{~mm}$. TheraCal LC is FDA approved as an appitite motivating liner with the capability to encourage apatite crystal formation, similar to commercially existing self curing MTA product. $.23,24$

\section{Conclusion}

Indirect pulp capping can be the safe and justified way to protect the pulp if proper case selection, adequate isolation, caries control, selection of suitable pulp capping material is possible to carry out. Clarity on the biology of caries, comprehension of the technological advances and conviction about improved restorative materials have initiated a pulp preservation that indeed is a boon to the clinician and the patient.

\section{References}

1. Mjor IA, Sveen OB, Heyeraas KJ. Pulp-dentin biology in restorative dentistry; Part 1: Normal structure and physiology. Quintessence Int. 2001; 32: $427-46$

2. Holland GR, Henry O, Rafter TM. Protecting the pulp and preserving the apex endodonticsprinciples and practice $4^{\text {th }}$ edn, chap- 2 .

3. Miyashita H, Worthington HV, Qualtrough A, Plasschaert A. Pulp management for caries in adults: Maintaining pulp vitality. Cochrane Database Syst Rev. 2007; 18: CD004484.

4. BjØrndl L, Mjör IA. Pulp-dentine biology in restorative dentistry. Part 4: Dental caries: Characteristics of lesions and pulpal reactions. Quintessence Int. 2001; 32: 717-36.

5. Dumsha T, Hovland E. Consideration and treatment of direct and indirect pulp capping, Dent Clin North Am. 1985; 29: 251-59.

6. Maltz M, Oliveira EF, Frontanella V, Bianchi R. A clinical, microbiological and radiological study of deep carious lesions after incomplete caries removal. Quintessence Int. 2002; 33: 151-59.

7. Fagundes TC, Barata THE, Prakki A, Bresciani E, Pereira JC. Indirect pulp treatment in the permanent molar, Case report of 4 years follow-up. Appl Oral Sci. 2009; 17: 70-74.

8. Bogen G, Chandler NP. Vital pulp therapy. In: Ingle's Endodontics. Ingle JI (ed). $6^{\text {th }}$ edn. Onterio, BC Decker Inc., 2008, pp 1110-30.
9. Auschill TM, Anweiler NB, Hellwig E, ZamaniAlaei A, Sculean A. Success rate of direct pulp capping with calcium hydroxide [in German]. Schweiz Monatsschr Zahnmed. 2003; 113: 946-52.

10. Dammaschke T. Biodentine ${ }^{\mathrm{TM}}$ : A new bioactive cement for direct pulp capping. No. 1. Seftodont, Case studies collection. 2012, pp 4-9.

11. BjØrndl L, Kidd EA. The treatment of deep dentine caries lesions. Dent Update. 2005; 32: 40213.

12. Fairbourn DR, Charbeneau GT, Loesche WJ. Effect of improved dycal and IRM on bacteria in deep carious lesions. JADA. 1980; 100: 547-52.

13. BjØrndl L. Dentin and pulp reaction to caries and operative treatment: Biological variables affecting treatment outcome. Endo Topics. 2002; 2: 10-23.

14. Murray PE, Godoy FG. The incidence of pulp healing defect with direct pulp capping materials. Am J Dent. 2006; 19: 171-77.

15. Estrela C, Holland R. Calcium hydroxide: Study based on scientific evidences. J Appl Oral Sci. 2003; 11: 269-82.

16. Wiener R. Liners, bases and cements: Material selection and clinical application. Dent Today. 2005; 64: 66-72.

17. Hilton TJ. Keys to clinical success with pulp capping: A review of the literature. Oper Dent. 2009; 34: 615-25.

18. Al-Araby A, Al-Jabab A. The effect of some dentin bonding agents on Dycal lining cement. Saudi Dent J. 2004; 16: 102-06.

19. Modena KC, Casas-Apayco LC, Atta MT, et al. Cytotoxicity and biocompatibility of direct and indirect pulp capping materials. J Appl Oral Sci. 2009; 17: 544-54.

20. Benoist FL, Ndiaye FG, Kane AW, Benoist HM, Farge P. Evaluation of mineral trioxide aggregate (MTA) versus calcium hydroxide (dycal) in the formation of a dentin bridge: A randomized controlled trial. Int Dent J. 2012; 62: 33-39.

21. Firla MT. Direct pulp capping with a bioactive dentin substitute: Biodentine ${ }^{\mathrm{TM}}$. No. 1 . Septodont, Case studies collection. 2012, pp 17-22.

22. Firla MT. Biodentine: A bioactive dentin substitute. IOSR-JDMS Rev. 2014; 13: 13-17.

23. Tran V, pradelle-Plasse N, Colon P. Microlikage of a new restorative calcium base cement (biodentine $^{\mathrm{TM}}$ ). Oral presentation Pep IADR, London. 2008.

24. Gandolfi MG, Siboni F, Taddei P, et al. Apatiteforming ability of TheraCal LC pulp-capping material. J Dent Res. 2011; 90(Spe A). Abstract 2520 . 